\title{
Polydiacetylene (PDA) liposome-based immune- sensor for the detection of exosomes
}

Changheon Kim, $\uparrow$ and Kangwon Lee*,,

$\dagger$ Program in Nanoscience and Technology, Graduate School of Convergence Science and

Technology, Seoul National University, Seoul, 08826 Republic of Korea 
(a)

(b)
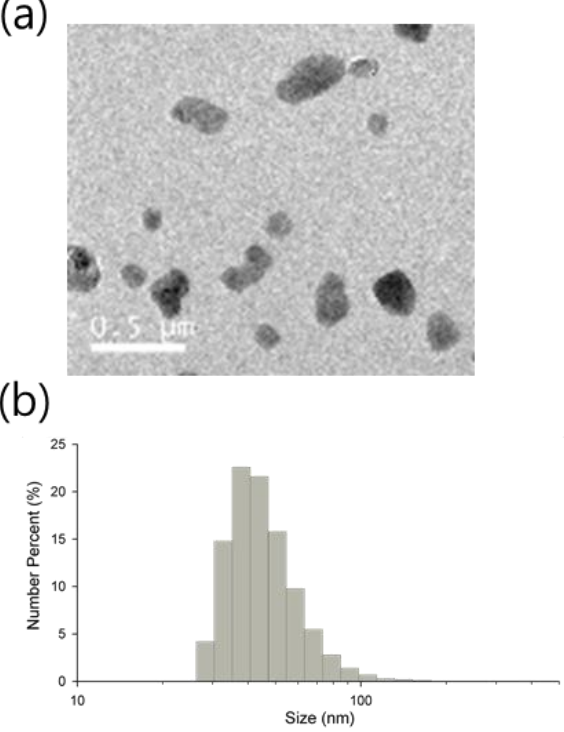

${ }_{E 7}(\mathrm{C})$

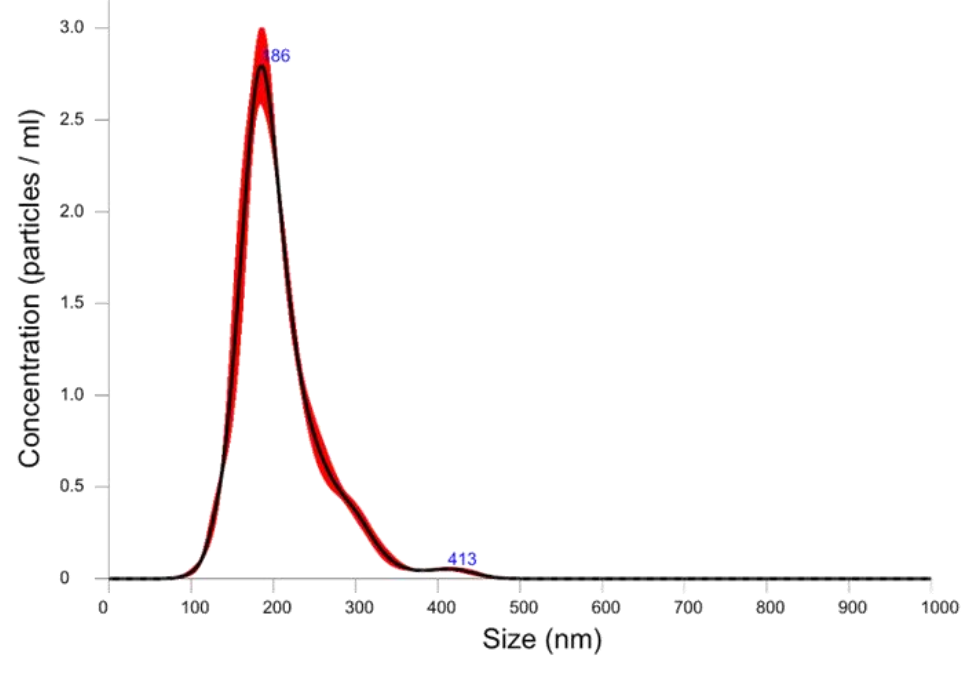

Figure S1. Characterization of isolated exosome from healthy human plasma. TEM image of exosomes $($ scale $=0.5 \mathrm{um})(\mathrm{a})$, size distribution of exosomes by DLS analysis (b) and exosome concentration of 100-fold diluted solution obtained from NTA data (c). 

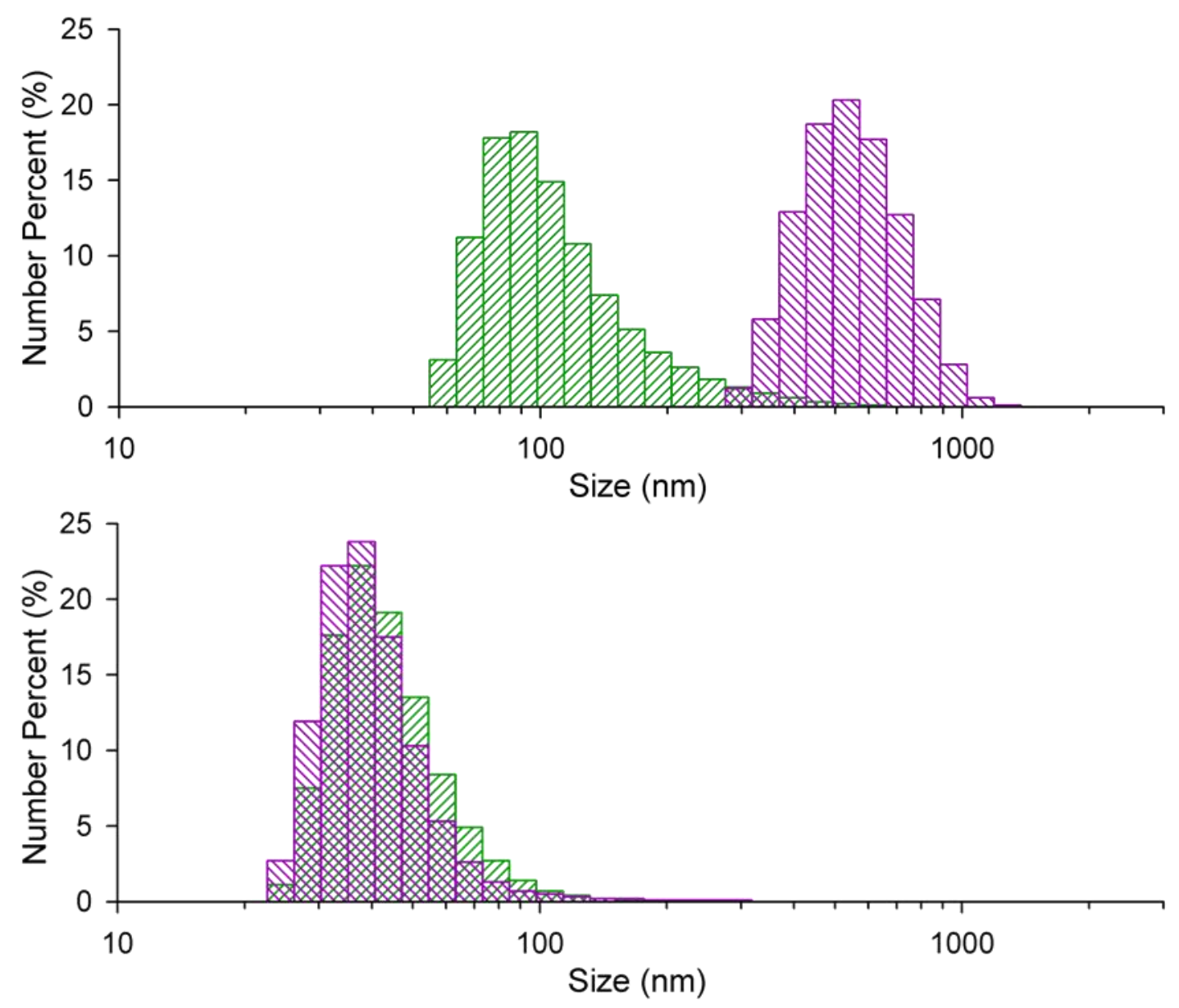

Figure S2. Changes in size distribution of PDA liposomes before and after EDC / NHS reaction. PCDA liposome (top), PCDA / DMPC liposome (bottom). 


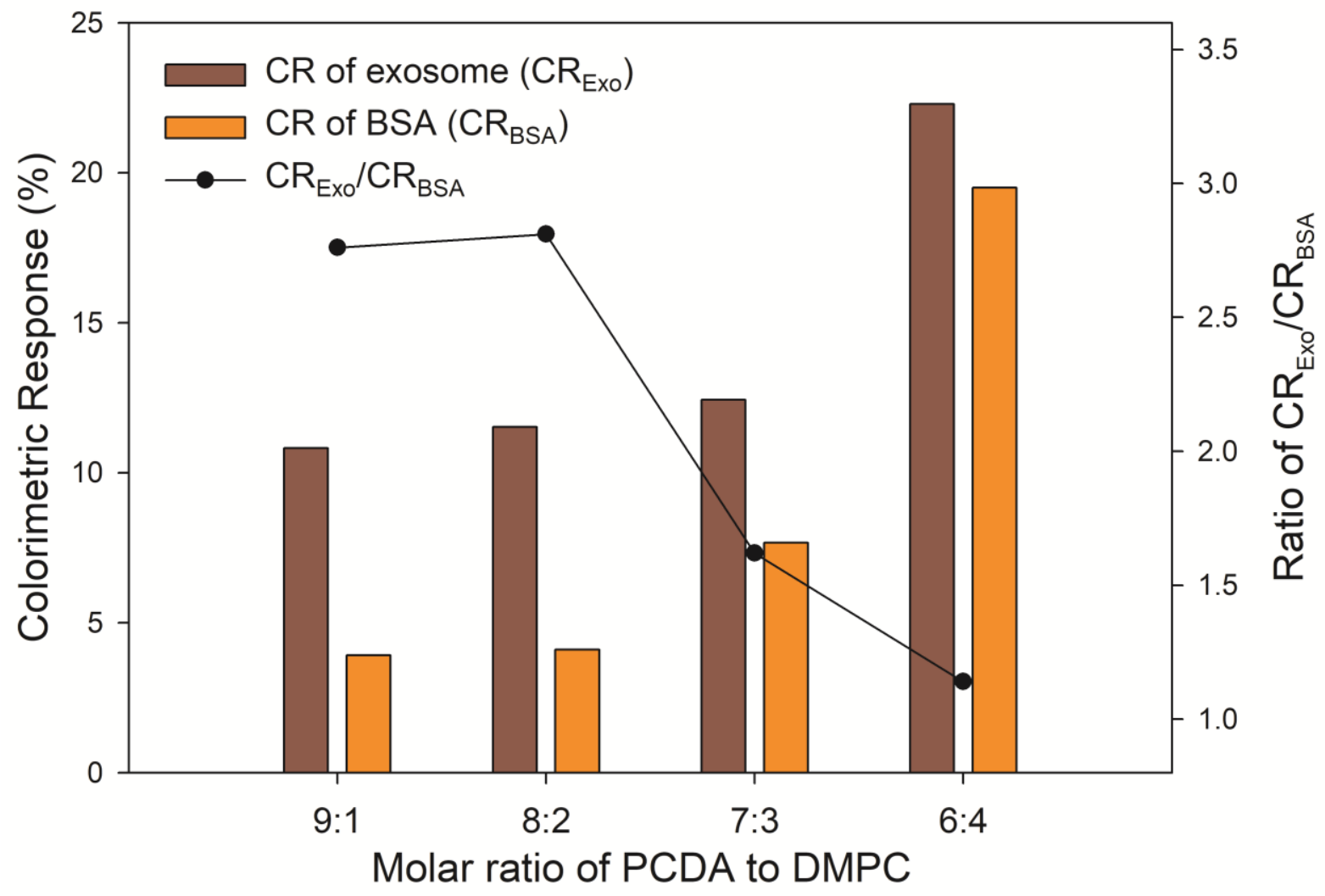

Figure S3. Colorimetric change by exosome (specific, $2 \times 10^{10}$ vesicles $/ \mathrm{mL}$ ) and Bovine serum albumin (non-specific, $0.1 \mathrm{mg} / \mathrm{mL}$ ) with the changes of PCDA and DMPC molar ratio 

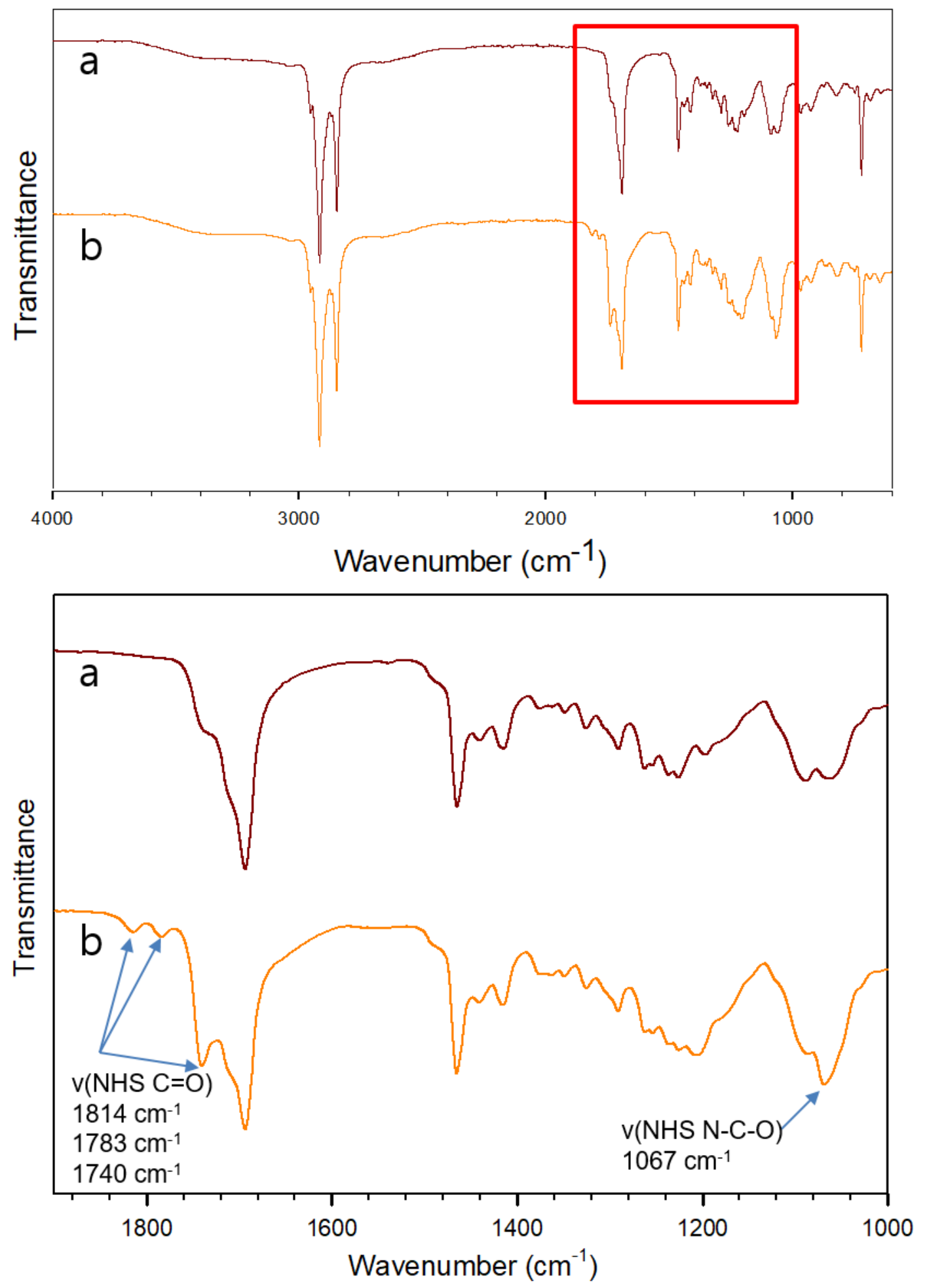

Figure S4. FT-IR spectra of PCDA/DMPC vesicle (a) and NHS-activated PCDA/DMPC vesicle (b). 

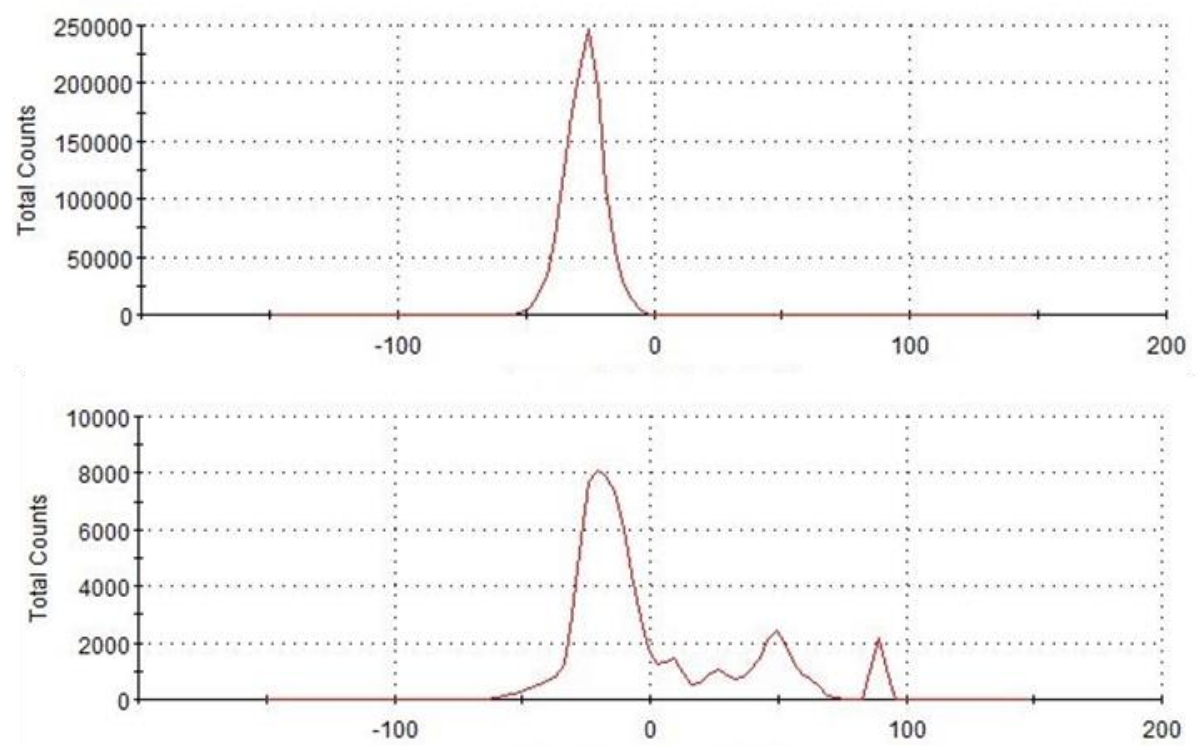

Figure S5. Zeta potential distribution of PCDA liposome (top) and anti CD-63 antibody attached PCDA liposome (bottom) 


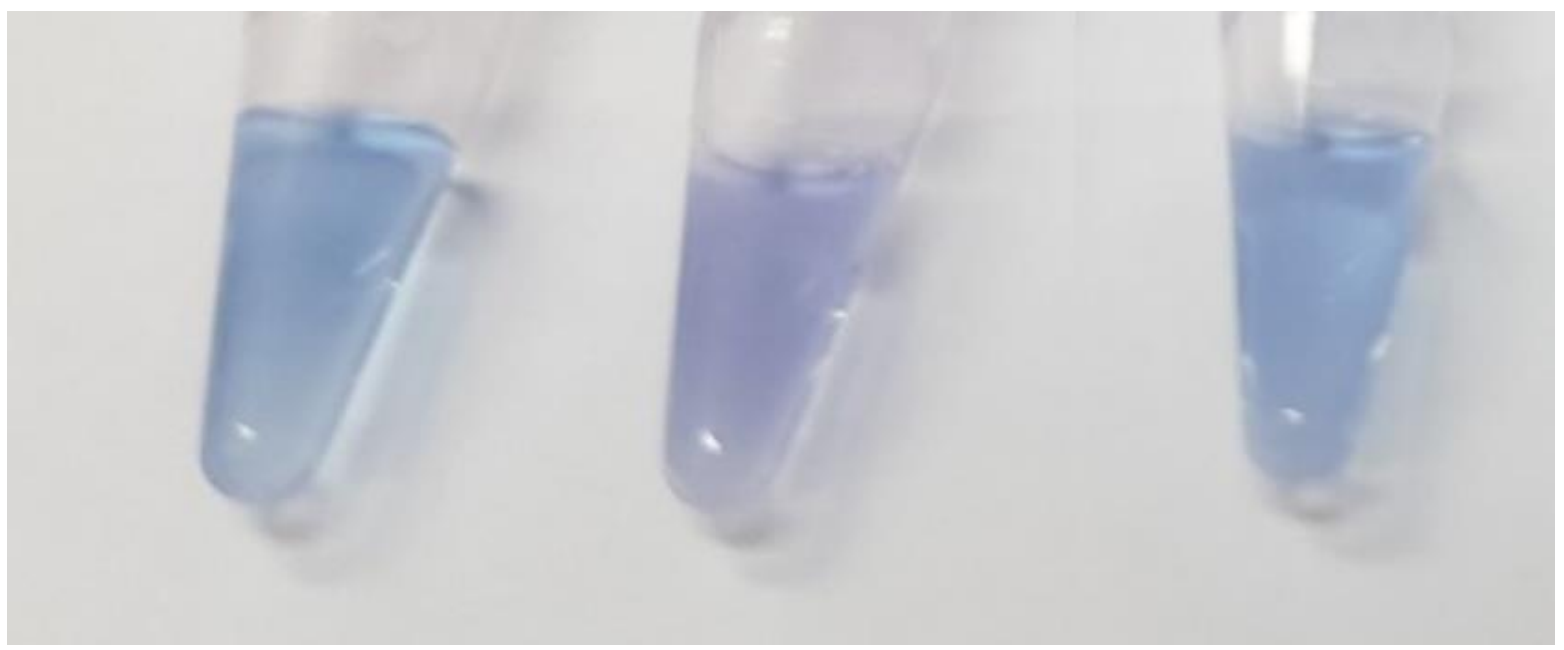

Figure S6. Colorimetric change of PDA Immunosensor with PBS solution (left), high concentration $\left(\mathbf{1 . 5} \times \mathbf{1 0}^{\mathbf{1 0}}\right.$ vesicles $\left./ \mathrm{ml}\right)$ exosome (middle) and low concentration $\left(\mathbf{7 . 5} \times \mathbf{1 0}^{\mathbf{7}}\right.$ vesicles/ml ) exosome (right) 

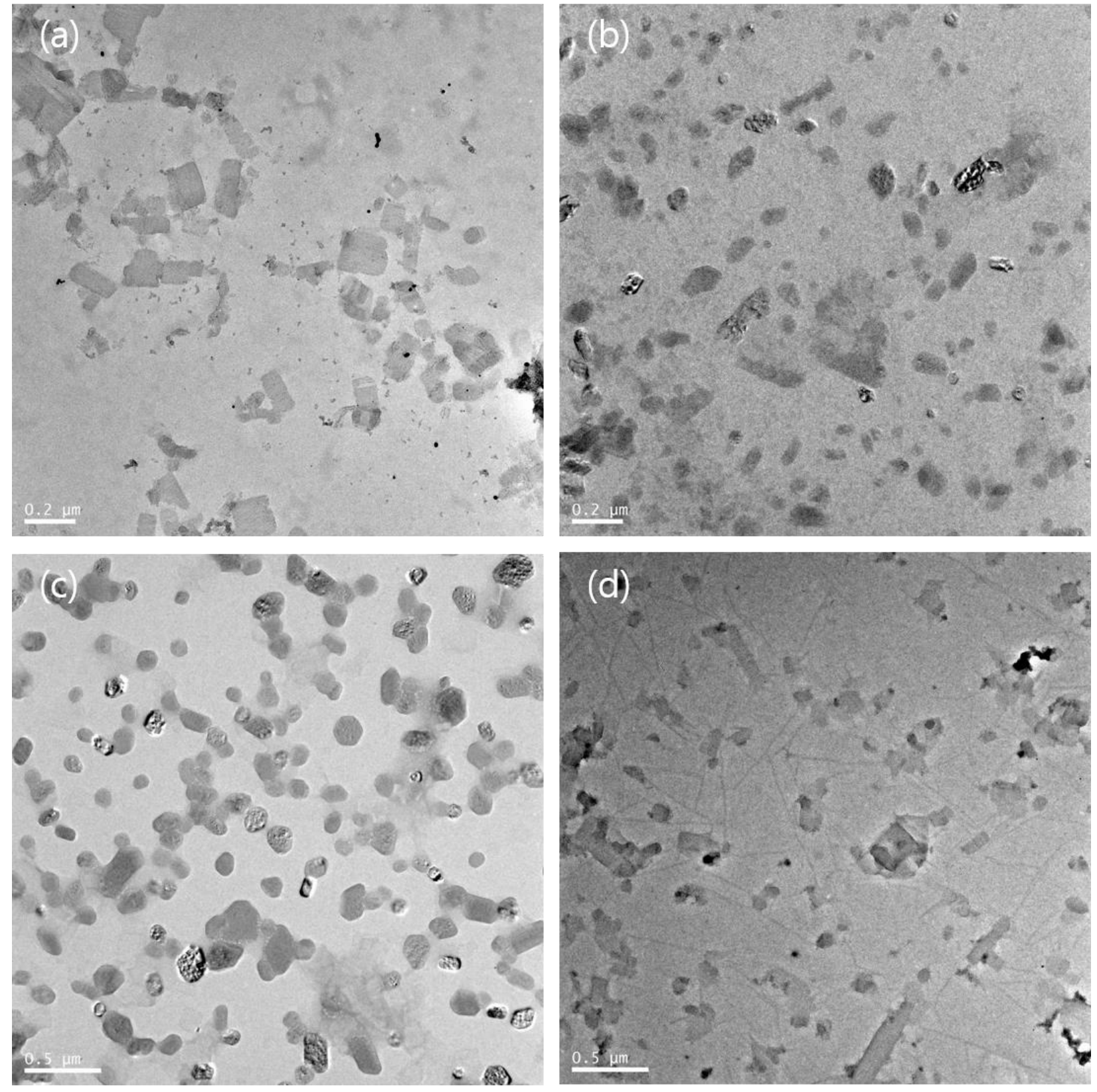

Figure S7. TEM image to confirm selectivity of PDA liposome sensor. PDA liposomes with exosomal tetraspanin receptor were added to BSA solution (a) and fibrinogen (b). PDA liposome blocked with BSA were added to exosome solution (c) and fibrinogen solution (d). 